\title{
The 2-Hydroxyiminostilbene Metabolite of Carbamazepine or the Supernatant from Incubation of Hepatocytes with Carbamazepine Activates Inflammasomes: Implications for Carbamazepine-Induced Hypersensitivity Reactions ${ }^{[\mathbb{S}}$
}

\author{
Received May 15, 2019; accepted July 10, 2019
}

\section{ABSTRACT}

Although the pathophysiology of carbamazepine-induced idiosyncratic or hypersensitivity reactions is unclear, they are presumed to be immune mediated, involving a complex interaction between drug metabolism and activation of the immune system. Cell stress can be caused by reactive metabolites, and this has the potential to release damage-associated molecular patterns (DAMPs), which are responsible for activation of the immune system. Idiosyncratic drug reactions occur mainly in the liver because of its role in drug metabolism and reactive metabolite formation. DAMPs can activate inflammasomes, which may be a common mechanism by which DAMPs lead to an immune response. In the present study, we investigated whether carbamazepine induces the release of DAMPs by using human hepatocarcinoma functional liver cell-4 (FLC-4) cells for bioactivation of carbamazepine. THP-1 cells, a human macrophage cell line, were used for detecting inflammasome activation. We found that increased caspase -1 activity and production of interleukin- $1 \beta$ by THP-1 cells were caused by the supernatant from the incubation of carbamazepine with FLC-4 cells. In the supernatant, heat shock protein 60 was significantly increased. In addition, 2-hydroxyiminostilbene, which is a metabolite of carbamazepine, activated inflammasomes. These results suggest that the reactive iminoquinone metabolite can directly activate inflammasomes or that stressed hepatocytes cause the release of DAMPs, which are responsible for inflammasome activation. The activation of inflammasomes may be an important step in the immune system activation by carbamazepine, which can lead to hypersensitivity reactions in some patients.

\section{SIGNIFICANCE STATEMENT}

A metabolite of carbamazepine, 2-hydroxyiminostilbene itself, and the damage-associated molecular patterns released from hepatocytes incubated with carbamazepine activated inflammasomes. The activation of inflammasomes may be an important step in the immune system activation by carbamazepine, which can lead to hypersensitivity reactions in some patients.

\section{Introduction}

Carbamazepine (5H-dibenzo[b,f]azepine-5-carboxamide) is currently recommended as the first line of therapy for the treatment of epilepsy in patients with partial-onset seizures (Marson et al., 2007). However, carbamazepine is associated with a significant risk of adverse reactions that primarily affect the skin but can also include other organs, such as the liver and bone marrow (Pellock, 1987; Pirmohamed et al., 2011). Although the mechanism of hypersensitivity reactions to carbamazepine is not well defined, it has been hypothesized to be immune-mediated, involving a complex interaction among drug metabolism, genetic variation in human leukocyte antigens (HLAs), and activation of the immune system (Yip et al., 2017). In most cases, the immune response appears to be initiated by a reactive metabolite (Cho and Uetrecht, 2017). Carbamazepine is extensively metabolized in the liver, and more than

This work was supported by the Japan Society for the Promotion of Science KAKENHI [JP18K06767].

The authors declare that they have no conflict of interest.

https://doi.org/10.1124/dmd.119.087981.

S This article has supplemental material available at dmd.aspetjournals.org.
30 metabolites have been identified in urine (Lertratanangkoon and Horning, 1982). 2-Hydroxycarbamazepine is one of the major metabolites, and it can be further metabolized to a reactive iminoquinone, which can redox cycle between the iminoquinone and 2-hydroxyiminostilbene (Ju and Uetrecht, 1999) (Fig. 1). Another potential reactive metabolite is carbamazepine-10,11-epoxide, an abundant and pharmacologically active metabolite of carbamazepine (Potter and Donnelly, 1998; Breton et al., 2005). It reacts with glutathione to form two isomeric carbamazepine10,11-epoxide-glutathione adducts in vitro and covalently binds to human liver microsomes (Bu et al., 2005).

The dominant and complementary hypotheses that have been proposed to explain how reactive metabolites induce the immune responses that result in idiosyncratic drug reactions are the hapten and danger hypotheses (Cho and Uetrecht, 2017). Reactive metabolites can generate drug-modified peptides that are presented in the context of HLA molecules (hapten hypothesis). However, a strong immune response is not induced by foreign proteins alone; thus, it requires a second or danger signal that is produced by costimulatory molecules on antigen presenting cells (APCs) (Matzinger, 1994). Cell damage can be caused by reactive metabolites, leading to the release of damage-associated molecular patterns (DAMPs). DAMPs act through receptors, such as

ABBREVIATIONS: ABT, 1-aminobenzotriazole; APC, antigen presenting cell; DAMP, damage-associated molecular pattern; FLC-4, functional liver cell-4; HLA, human leukocyte antigen; HSP, heat shock protein; IL, interleukin; S100A8, S100 calcium-binding protein A8; S100A9, S100 calciumbinding protein A9. 


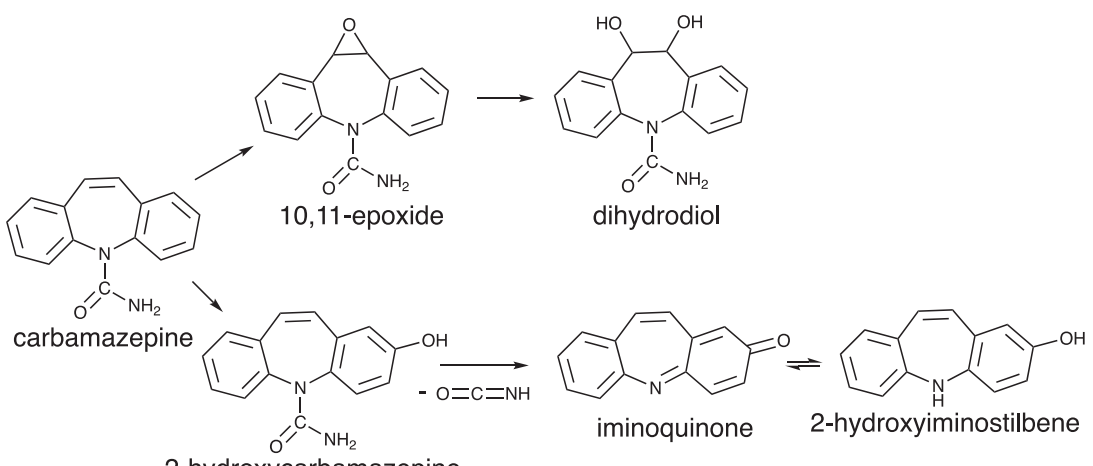

Fig. 1. Two bioactivation pathways of carbamazepine. Carbamazepine is oxidized by multiple cytochrome P450s to several metabolites, two major metabolites being the 10,11-epoxide and 2 -hydroxycarbamazepine. The 10,11-epoxide is weakly chemically reactive and is also hydrolyzed to the dihydrodiol. 2Hydroxycarbamazepine can be further oxidized to a reactive iminoquinone with the loss of isocyanate. This iminoquinone can react with protein thiols or be reduced nonenzymatically to 2-hydroxyiminostilbene. 2-Hydroxyiminostilbene can also be readily reoxidized to the iminoquinone by relatively mild oxidants without the requirement of cytochrome P450s. the receptor for advanced glycation end products and toll-like receptors. Inflammasome activation can be induced through these receptors, and this may be a common mechanism leading to APC activation by DAMPs (Bettigole and Glimcher, 2015). We tested the ability of the culture supernatant of hepatocytes treated with carbamazepine to activate differentiated THP-1 cells, a human macrophage cell line. In addition, we tested the ability of two carbamazepine metabolites, 2-hydroxyiminostilbene and the 10,11-epoxide, to directly activate inflammasomes in differentiated THP-1 cells.

\section{Materials and Methods}

Reagents. Carbamazepine and 1-aminobenzotriazole (ABT) were provided by Tokyo Chemical Industry Co., Ltd (Tokyo, Japan). 2-Hydroxyiminostilbene and carbamazepine-10,11-epoxide were provided by Toronto Research Chemicals (ON, Canada). The other reagents used in the study were of extra-pure grade.
Cell Cultures. Functional liver cell-4 (FLC-4) cells (JCRB0435; Health Science Research Resources Bank, Osaka, Japan) and THP-1 cells (JCRB0112; Japanese Collection of Research Bioresources Cell Bank, Osaka, Japan) were used in this study. Cell culture was performed following our previous study (Kato and Uetrecht, 2017). The carbamazepine concentrations were within their therapeutic concentrations $(10-100 \mu \mathrm{M})$ (Bertilsson and Tomson, 1986). Although there is no change in cell viability between 10 and $100 \mu \mathrm{M}$ carbamazepine and carbamazepine-10,11-epoxide, cell viability at $100 \mu \mathrm{M}$ 2-hydroxyiminostilbene significantly decreased. Therefore, the concentration of 2-hydroxyiminostilbene used in this study was set at 3-30 $\mu \mathrm{M}$. A detailed description of the quantification of carbamazepine and its metabolites is provided in the Supplemental Material. ABT $(1 \mathrm{mM})$ was used as an inhibitor of cytochrome P450 activities, and Ac-YVAD-CHO (1 $\mu \mathrm{M}$; Promega Corporation, Madison, WI) was used as an inhibitor of caspase-1 activity. In addition, heat shock protein 60 (HSP60) rabbit polyclonal antibody (1:1000, 15282-1-AP; Proteintech Group, Inc., Rosemont, IL) was added to the supernatant of FLC-4 cells with carbamazepine for 7 days.

\section{B Hepatocyte supernatant added to THP-1 cells}

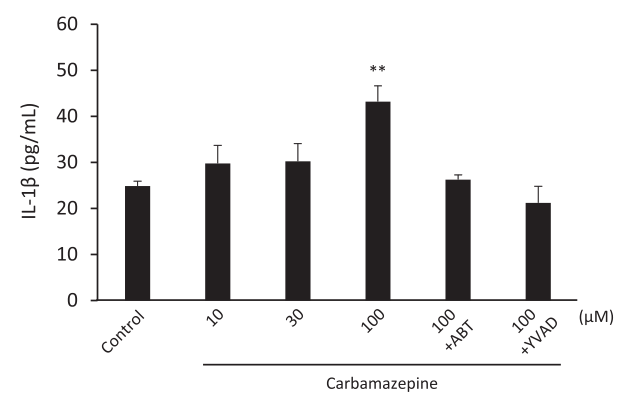

D Hepatocyte supernatant added to THP-1 cells

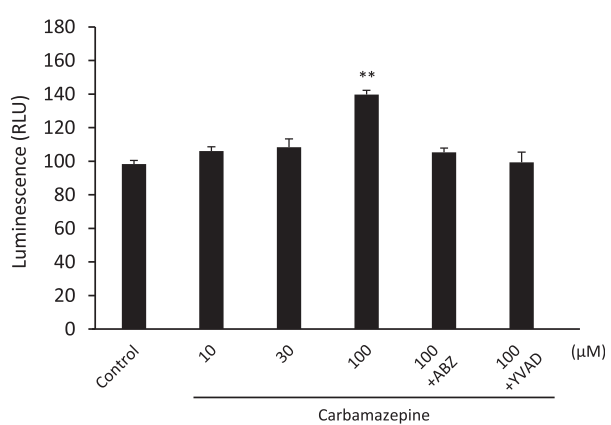

Fig. 2. THP-1 cells incubated with 2-hydroxyiminostilbene or the supernatant of FLC-4 cells with carbamazepine led to release of IL-1 $\beta$ and an increase in caspase-1 activity. (A) IL-1 $\beta$ secretion by differentiated THP-1 cells, which were treated with carbamazepine, carbamazepine-10,11-epoxide, or 2-hydroxyiminostilbene for 24 hours. (B) IL-1 $\beta$ produced by incubation of differentiated THP-1 cells, which were treated with the supernatant of FLC-4 cells, with carbamazepine for 7 days, with or without a cytochrome P450 inhibitor (ABT) or a caspase-1 inhibitor [Ac-YVAD-CHO (YVAD)]. Caspase-1 activity of differentiated THP-1 cells, which were treated with 2hydroxyiminostilbene (C) or the supernatant from hepatocytes with carbamazepine (D), with or without YVAD, for 24 hours. Statistical comparisons were performed using the Tukey multiple comparison tests. A $P$ value $<0.05$ was considered to be statistically significant $(* * P<0.01, n=3-5)$. RLU, relative light unit. 
A

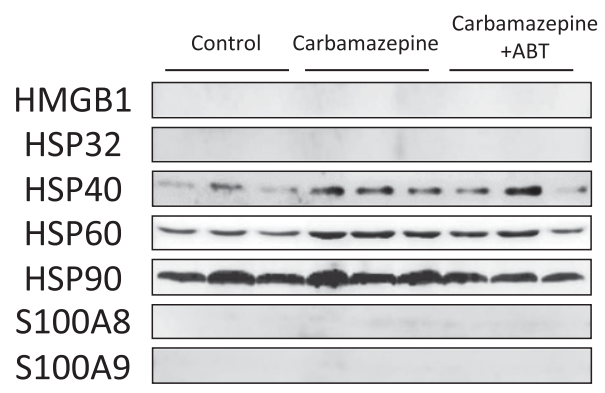

C

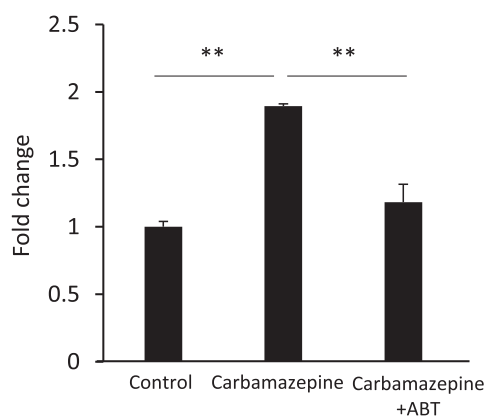

B

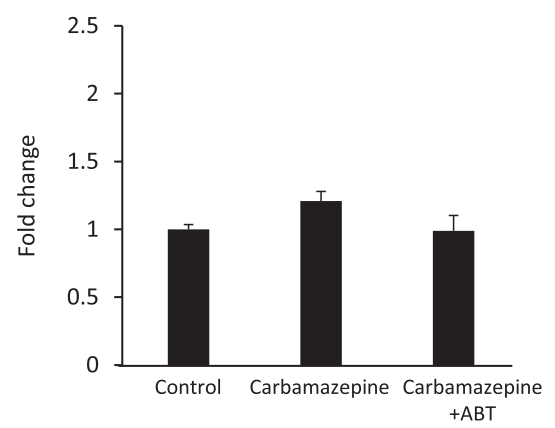

D

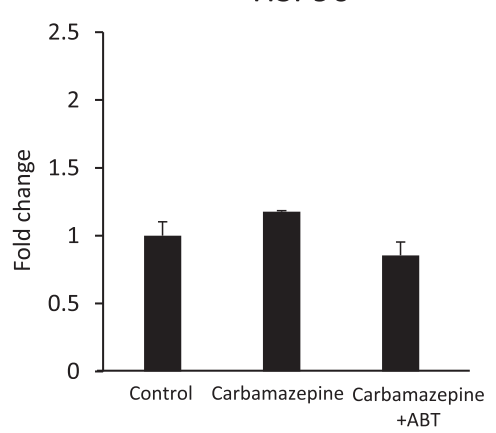

E

Fig. 3. HSP60 was released from hepatocytes as DAMPs. (A) Western blot analysis of proteins in culture medium [high mobility group box 1 (HMGB1), HSP32, HSP40, HSP60, HSP90, S100A8, and S100A9] that were released into the supernatant from incubation of hepatocytes with carbamazepine incubated for 7 days with carbamazepine $(100 \mu \mathrm{M})$, and their quantitative analysis of HSP40 (B), HSP60 (C), and HSP90 (D). (E) IL-1 $\beta$ secreted by differentiated THP-1 cells, which were treated with carbamazepine, with or without anti-HSP60 antibody (HSP60Ab) for 24 hours. HSP60 rabbit polyclonal antibody was added to the supernatant from the FLC-4 cells, which had been cultured with carbamazepine for 7 days. Statistical comparisons were performed using the Tukey multiple comparison test. A $P$ value $<0.05$ was considered to be statistically significant $(* * P<0.01, n=3)$.

Western Blotting. Supernatants from FLC-4 cells incubated with carbamazepine $(20 \mu \mathrm{l})$ were loaded and separated by electrophoresis on an $8 \%$ sodium dodecyl sulfate polyacrylamide gel and transferred onto a polyvinylidene difluoride membrane $(0.45 \mu \mathrm{m}$; Merck KGaA, Darmstadt, Germany). High mobility group box-1 rabbit polyclonal antibody (10829-1-AP; Proteintech Group, Inc.), HSP32 rabbit polyclonal antibody (10701-1-AP; Proteintech Group, Inc.), HSP40 rabbit polyclonal antibody (13174-1-AP; Proteintech Group, Inc.), HSP60 rabbit polyclonal antibody (15282-1-AP; Proteintech Group, Inc.), HSP90 rabbit polyclonal antibody (13171-1-AP; Proteintech Group, Inc.), S100 calciumbinding protein A8 (S100A8) rabbit polyclonal antibody (GTX54721; GeneTex, Inc., Irvine, CA), and S100 calcium-binding protein A9 (S100A9) rabbit polyclonal antibody (GTX129575; GeneTex, Inc.) were used as the primary antibody and were detected by goat anti-rabbit IgG-peroxidase (Santa Cruz Biotechnology, Dallas, TX). After washing, immunoreaction was detected by using Luminata Classico Western HRP Substrates (Merck KGaA).

Interleukin-1 $\beta$ Concentration in Culture Medium. Fifty microliters of culture medium was collected from differentiated THP-1 cells, and interleukin- $1 \beta$ (IL-1 $\beta$ ) concentration was quantified by using an ELISA kit (BioLegend, Inc., San Diego, CA).

Caspase-1 Activity of Differentiated THP-1 Cells. Caspase-1 activity of differentiated THP-1 cells was measured by using the Caspase-Glo 1 Inflammasome Assay (Promega Corporation). The reagent was added to each well and then incubated for 1 hour at room temperature. The luminescence was measured with a plate reader.

Data Analysis. Statistical comparisons were performed using the Tukey multiple comparison tests. A $P$ value $<0.05$ was considered to be statistically significant. Data are expressed as the mean \pm S.D.

\section{Results and Discussion}

In our previous studies, it was reported that three-dimensional cultures of a human FLC-4 cell line synthesized and secreted liver-specific proteins (albumin, $\alpha$-fetoprotein, etc.). These cells also have a high capacity to metabolize drugs and were used to produce reactive metabolites of carbamazepine (Kato et al., 2014). In the hepatocyte supernatant, carbamazepine was decreased $(0.1-0.048 \mathrm{mM})$, and carbamazepine10,11-epoxide $(1 \mu \mathrm{M})$ and 2-hydroxyiminostilbene $(0.26 \mu \mathrm{M})$ were detected in the hepatocyte supernatant with 7-day incubation of carbamazepine (Supplemental Fig. 1). In addition, there was a metabolite with an $\mathrm{m} / \mathrm{z} 253$, consistent with 2- and/or 3-hydroxycarbamazepine. Given that the epoxide is chemically reactive and 2-hydroxyiminostilbene is a product of the reactive iminoquinone, undoubtedly the metabolism through these pathways was more extensive than indicated by the observed metabolites. We found that THP-1 cells were not activated by carbamazepine or carbamazepine-10,11-epoxide, but they were activated by 2 -hydroxyiminostilbene, leading to the release of IL- $1 \beta$ and production of active caspase-1 (Fig. 2A). However, the culture supernatant from FLC-4 hepatocytes with carbamazepine did lead to the activation of inflammasomes in THP-1 cells (Fig. 2B). IL-1 $\beta$ production was inhibited by the addition of the cytochrome $\mathrm{P} 450$ inhibitor (ABT). In addition, 2-hydroxyiminostilbene and the supernatant of FLC- 4 cells increased caspase- 1 activity in differentiated THP-1 cells (Fig. 2, C and D).

We also identified DAMPs in the culture supernatant of hepatocytes. Higuchi et al. (2012) reported that the mRNA expression of S100A8 and S100A9 significantly increased in mice with carbamazepineinduced liver injury. However, in the Higuchi et al. study, a very high dose of carbamazepine was used; this induced acute toxicity, which is presumably not immune-mediated. In our study, although S100A8 and S100A9 proteins were not detected, HSP60 was significantly increased in the culture supernatant of hepatocytes treated with 
carbamazepine (Figs. 2 and 3). The cytochrome P450 inhibitor, ABT, prevented this increase in HSP60. Furthermore, the supernatant from hepatocytes cultured with carbamazepine and treated with an anti-HSP60 antibody did not increase IL- $1 \beta$ production in THP-1 cells, which suggests that HSP60 activates the inflammasome. It has been reported that HSP60 mediates IL- $1 \beta$ production by regulating the NLRP3 inflammasome pathway (Swaroop et al., 2018). Therefore, the release of HSP60 from hepatocytes caused by reactive metabolites of carbamazepine may be responsible for the activation of inflammasomes in differentiated THP-1 cells.

Additionally, signal 2, which is produced by costimulatory molecules on APCs, is required to induce an immune response (danger hypothesis) (Matzinger, 1994). The results of this study are consistent with the working hypothesis that the mechanism of carbamazepineinduced hypersensitivity reactions involves oxidation of carbamazepine in the liver to the iminoquinone of 2-hydroxyiminostilbene. This can covalently bind to proteins in the liver, causing the release of DAMPS. It can also be reduced to 2-hydroxyiminostilbene and reach the blood. In other tissues, the 2-hydroxyiminostilbene can be reoxidized to the iminoquinone by peroxidases and activate inflammasomes there. Reactive metabolites may activate the inflammasome indirectly by inducing the release of DAMPs, or may directly activate inflammasomes. The activated inflammasome results in caspase-1-mediated conversion of pro-IL- $1 \beta$ into its bioactive mature form, IL- $1 \beta$. IL- $1 \beta$ is a strong proinflammatory molecule capable of promoting an immune response (Guo et al., 2015). Therefore, the activation of inflammasomes may be an important bridge between reactive metabolite formation and the induction of an immune response. In some patients with the right HLA/T cell receptor combination, this activation can lead to a hypersensitivity reaction.

An independent piece of information also argues against involvement of the 10,11-epoxide in the mechanism of carbamazepine-induced hypersensitivity reactions. Oxcarbazepine also causes hypersensitivity reactions similar to those of carbamazepine. The mechanism must be similar because oxcarbazepine-induced toxic epidermal necrolysis is associated with the same HLA genotype as toxic epidermal necrolysis caused by carbamazepine (Chen et al., 2017). That implies that the drug-modified protein binds with a similar orientation. Oxcarbazepine has the potential to form an iminoquinone similar to that of carbamazepine, but it cannot form a 10,11epoxide. However, we cannot rule out the possibility that the epoxide or other metabolites contribute to the release of DAMPs from hepatocytes.

In conclusion, our results support the hypothesis that idiosyncratic or hypersensitivity reactions associated with carbamazepine involve oxidation of carbamazepine into reactive metabolites in hepatocytes, the most likely being the iminoquinone. This results in the production of DAMPs that activate inflammasomes, thereby resulting in an immune response. This method may provide a method to study the mechanism of idiosyncratic drug reactions and even predict which drug candidates are likely to cause such adverse reactions.
Department of Cardiovascular Pharmacotherapy and Toxicology, Osaka University of Pharmaceutical Sciences, Osaka, Japan (R.K., Y.I., T.H.) and Department of Pharmaceutical Sciences, Leslie Dan Faculty of Pharmacy, University of Toronto, Toronto, Ontario, Canada (J.U.)

\section{Authorship Contributions}

Participated in research design: Kato, Uetrecht. Conducted experiments: Kato.

Performed data analysis: Kato, Ijiri, Hayashi, Uetrecht.

Wrote or contributed to the writing of the manuscript: Kato, Uetrecht.

\section{References}

Bertilsson L and Tomson T (1986) Clinical pharmacokinetics and pharmacological effects of carbamazepine and carbamazepine-10,11-epoxide. An update. Clin Pharmacokinet 11:177-198 Bettigole SE and Glimcher LH (2015) Endoplasmic reticulum stress in immunity. Аnnu Rev Immunol 33:107-138.

Breton H, Cociglio M, Bressolle F, Peyriere H, Blayac JP, and Hillaire-Buys D (2005) Liquid chromatography-electrospray mass spectrometry determination of carbamazepine, oxcarbazepine and eight of their metabolites in human plasma. J Chromatogr B Analyt Technol Biomed Life Sci 828:80-90.

Bu HZ, Kang P, Deese AJ, Zhao P, and Pool WF (2005) Human in vitro glutathionyl and protein adducts of carbamazepine-10,11-epoxide, a stable and pharmacologically active metabolite of carbamazepine. Drug Metab Dispos 33:1920-1924.

Chen CB, Hsiao YH, Wu T, Hsih MS, Tassaneeyakul W, Jorns TP, Sukasem C, Hsu CN, Su SC, Chang WC, et al.; Taiwan Severe Cutaneous Adverse Reaction Consortium (2017) Risk and association of HLA with oxcarbazepine-induced cutaneous adverse reactions in Asians. Neurology 88:78-86.

Cho T and Uetrecht J (2017) How reactive metabolites induce an immune response that sometimes leads to an idiosyncratic drug reaction. Chem Res Toxicol 30:295-314.

Guo H, Callaway JB, and Ting JP (2015) Inflammasomes: mechanism of action, role in disease, and therapeutics. Nat Med 21:677-687.

Higuchi S, Yano A, Takai S, Tsuneyama K, Fukami T, Nakajima M, and Yokoi T (2012) Metabolic activation and inflammation reactions involved in carbamazepine-induced liver injury. Toxicol Sci 130:4-16.

Ju C and Uetrecht JP (1999) Detection of 2-hydroxyiminostilbene in the urine of patients taking carbamazepine and its oxidation to a reactive iminoquinone intermediate. J Pharmacol Exp Ther 288:51-56. Kato R, Shigemoto K, Akiyama H, Ieda A, Ijiri Y, and Hayashi T (2014) Human hepatocarcinoma functional liver cell-4 cell line exhibits high expression of drug-metabolizing enzymes in threedimensional culture. Biol Pharm Bull 37:1782-1787.

Kato R and Uetrecht J (2017) Supernatant from hepatocyte cultures with drugs that cause idiosyncratic liver injury activates macrophage inflammasomes. Chem Res Toxicol 30:1327-1332. Lertratanangkoon K and Horning MG (1982) Metabolism of carbamazepine. Drug Metab Dispos 10:1-10.

Marson AG, Al-Kharusi AM, Alwaidh M, Appleton R, Baker GA, Chadwick DW, Cramp C Cockerell OC, Cooper PN, Doughty J, et al.; SANAD Study group (2007) The SANAD study of effectiveness of carbamazepine, gabapentin, lamotrigine, oxcarbazepine, or topiramate for treatment of partial epilepsy: an unblinded randomised controlled trial. Lancet 369:1000-1015. Matzinger P (1994) Tolerance, danger, and the extended family. Annu Rev Immunol 12:991-1045. Pellock JM (1987) Carbamazepine side effects in children and adults. Epilepsia 28 (Suppl 3):S64-S70. Pirmohamed M, Friedmann PS, Molokhia M, Loke YK, Smith C, Phillips E, La Grenade L, Carleton B, Papaluca-Amati M, Demoly P, et al. (2011) Phenotype standardization for immunemediated drug-induced skin injury. Clin Pharmacol Ther 89:896-901.

Potter JM and Donnelly A (1998) Carbamazepine-10,11-epoxide in therapeutic drug monitoring. Ther Drug Monit 20:652-657.

Swaroop S, Mahadevan A, Shankar SK, Adlakha YK, and Basu A (2018) HSP60 critically regulates endogenous IL-1 $\beta$ production in activated microglia by stimulating NLRP3 inflammasome pathway [published correction appears in J Neuroinflammation (2018) 15:317]. J Neuroinflammation 15:177.

Yip VLM, Meng X, Maggs JL, Jenkins RE, Marlot PT, Marson AG, Park BK, and Pirmohamed M (2017) Mass spectrometric characterization of circulating covalent protein adducts derived from epoxide metabolites of carbamazepine in patients. Chem Res Toxicol 30:1419-1435.

Address correspondence to: Dr. Ryuji Kato, Department of Cardiovascular Pharmacotherapy and Toxicology, Osaka University of Pharmaceutical Sciences, 4-20-1 Nasahara, Takatsuki, Osaka 569-1094, Japan. E-mail: rkato@gly.oups.ac.jp 